\title{
LOAD PREDICTION OF MICROGRID OPTIMAL OPERATION BASED ON IMPROVED ALGORITHM IN MACHINE LEARNING
}

\author{
Tao Yao ${ }^{1}$, Dan Jiang ${ }^{1}$, Rui Xin ${ }^{1}$, Junying $W u^{1}$, Sisi Sun ${ }^{1}$ \\ ${ }^{1}$ State Grid Hebei Information \& Telecommunication Branch, Shijiazhuang, Hebei 050000, China \\ E-mail:yaotedu@yeah.net
}

\begin{abstract}
Load prediction is an important problem in microgrid operation. In this study, the kernel extreme learning machine (KELM) algorithm in machine learning was used for load prediction. In order to improve the prediction accuracy, the KELM algorithm was improved by combining with Ant Colony Optimization (ACO) algorithm and Particle Swarm Optimization (PSO) algorithm, and then experiments were carried out on the property load of two communities in May-July. The experimental results showed that MAPE of the improved algorithm in predicting the load of the two communities was $1.43 \%$ and $1.57 \%$ respectively, and the operation time was $215 \mathrm{~s}$ and $223 \mathrm{~s}$ respectively, which were better than than the support vector machine (SVM) and KELM algorithms; the prediction results were close to the actual values, and the error changes were stable, which verified the effectiveness of the improved algorithm. The experimental results make some contributions to improve the accuracy of load prediction and promote the optimal operation of microgrid, which is conducive to the further development of microgrid.
\end{abstract}

Keywords: Machine Learning, Micro Grid, Load Prediction, Kernel Extreme Learning Machine, Swarm Intelligence Algorithm.

\section{Introduction}

With the further aggravation of energy shortage and environmental pollution [1], the development and utilization of new energy has become an inevitable choice. Microgrid is an effective way to use solar energy, wind energy and other energy [2], and it is a power generation and distribution system with a capacity less than or equal to $500 \mathrm{~kW}$ [3], which can realize distributed energy scheduling [4], fully meet the user's electricity demand, effectively improve the energy utilization efficiency, and has a very important value in improving the power quality and ensuring the reliability of power supply. It has broad prospects for development. The energy management of microgrid plays an important role in the efficient operation of microgrid. Load prediction is an important part of energy management, which has a direct impact on the planning, quality and trading of microgrid power supply, and it has been widely concerned by researchers. Zhang et al. [5] optimized the grey prediction model by using invasive weeds to solve the problem of low accuracy and verified through experiments that the method had small error. Chang et al. [6] proposed a combined prediction model optimized by particle swarm optimization algorithm, compared it with a single model, and verified its reliability in power load prediction. Jiani et al. [7] designed a hybrid prediction model combining empirical mode decomposition, cuckoo search algorithm and wavelet neural network, carried out experiments on halfhour power load in New South Wales, Australia, and found that the prediction results were very similar to the actual results. Chitzaz et al. [8] trained the self recursive wavelet neural network through Levenberg Marquardt (LM) learning algorithm and verified the effectiveness of the method through experiments. At present, there are many researches on the short-term prediction of solar and wind power generation and few researches on the shortterm prediction of micro grid load. This study analyzed the load prediction with machine algorithm, designed a kernel extreme learning machine (KELM) model combining Ant Colony Optimization (ACO) algorithm and Particle Swarm Optimization (PSO) algorithm, and verified the advantages of the method in time and accuracy through experiments, which was conducive to the optimal operation of microgrid and the further improvement of power supply reliability.

\section{Micro Grid Load Prediction}

Load prediction refers to predicting the load based on a large number of historical data according to the known power system and other situations. It can be divided into ultra short-term, short-term, mediumterm and long-term prediction according to the length of time. In order to obtain high-precision prediction results, it is necessary to master the historical data comprehensively and use advanced 
technical means and theories to forecast in order to reduce the prediction errors as much as possible. In the load prediction, the causes of errors may include data mutation, missing and other phenomena in the collection of historical data and large fluctuations in the load data induced by the occurrence of major events, meteorological conditions mutation or system failure and other events. In microgrid, the factors influencing load fluctuation include:

(1) climate conditions: temperature and precipitation have a direct impact on electrical loads such as cooling, heating and irrigation. Taking the high temperature in summer in the north and south as an example, the cooling power consumption of air conditioning in the south is significantly higher than that in the south; when the precipitation is rich, the farmland irrigation load will also be significantly lower than that in the period when the precipitation is little.

(2) economic conditions: in areas with high level of regional economic development, the power load of the tertiary industry is higher; the higher the degree of residents' electrification, the greater the difference of power load between peak and valley.

(3) emergencies: unpredictable factors such as sudden failure and maintenance of power grid, unexpected power rationing and so on will have a great impact on load data.

Improving the quality of load prediction has always been the focus and difficulty of researchers. At present, the commonly used methods include time series method, regression analysis method [9], similar day method, grey prediction method [10], artificial neural network, etc., and machine learning methods such as support vector machine (SVM) [11] also have good applications.

\section{KELM}

Compared with SVM, the extreme learning machine (ELM) [12] has advantages of high training speed, high accuracy and good performance in various fields. KELM is the derivation of the traditional ELM algorithm and the combination of kernel function and ELM; therefore it has better generalization performance and high calculation speed than ELM. Its specific principle is as follows:

The neural network function of ELM can be expressed as: $f(x)=h(x) \alpha$.

In the ELM function, the accuracy is guaranteed by minimizing the output error, i.e., $\lim _{L \rightarrow \infty}\left\|f(x)-f_{o}(x)\right\|$, where $L$ indicates the number of hidden layer nodes and $f_{o}(x)$ represents the function to be predicted.

Moreover output weight $\alpha$ is minimized, normal

number $C$ is introduced, and $\alpha=H^{T}\left(\frac{1}{C}+H H^{T}\right)^{-1} O$ is solved, where $H$ is a hidden layer matrix and $O$ is the predicted target value vector.

Combined with kernel function, KELM can be expressed as:

$$
\begin{aligned}
& f(x)=h(x) H^{T}\left(\frac{1}{C}+H H^{T}\right)^{-1} O=\left(\begin{array}{c}
K\left(x, x_{1}\right) \\
\vdots \\
K\left(x, x_{N}\right)
\end{array}\right)\left(\frac{1}{C}+\Psi_{E L M}\right)^{-1} O \\
& \Psi_{E L M(i, j)}=\exp \left(-\omega\left\|x_{i}-x_{j}\right\|^{2}\right)
\end{aligned}
$$

where $\Psi_{E L M}$ refers to a kernel matrix, $K\left(x_{i}, x_{j}\right)$ represents a kernel function, and $N$ stands for the mantissa of the input layer.

In KELM, $\Psi_{E L M}$ replaces random matrix $H$, which effectively enhances the stability of the model, but in KELM, the value of parameter $(C, \omega)$ will affect the prediction performance, which needs to be processed by optimization solution.

\section{Swarm Intelligence Improved KELM Algorithm \\ Algorithm}

Swarm intelligence algorithm comes from the simulation of biological behavior in nature, mainly including ant colony optimization algorithm (ACO) and particle swarm optimization algorithm (PSO). In this study, ACO and PSO are combined to realize the optimization of KELM parameters.

In ACO algorithm, the ant colony finds the shortest path to the food source through the perception of pheromone and transfers with transfer probability $P_{i j}^{k}(t)$, and $P_{i j}^{k}(t)$ is in direct proportion to heuristic factor $\eta_{i j}$. The formula of pheromone updating on the ant colony path is: $\mu_{i j}(t+n)=(1-\delta) \times \mu_{i j}(t)+\Delta \mu_{i j}$, where $\delta$ is the pheromone volatility and $\Delta \mu_{i j}$ represents the pheromone increment of this path.

In PSO algorithm, each particle, that is, each bird, represents a potential solution, and each particle has a speed, which is adjusted according to the movement of itself and other examples to achieve optimization. Suppose that in the D-dimensional search space, the number of particles is $N$, the best position $P_{\text {best }}$ of the $i$-th particle is $P_{i}=\left(P_{i 1}, P_{i 2}, \cdots, P_{i d}\right)$, the global extreme value is $g_{\text {best }}$, then the updating formulas of particle speed $V$ and position $X$ are:

$V_{i d}^{k+1}=w V_{i d}^{k}+c_{1} r_{1}\left(P_{i d}^{k}-X_{i d}^{k}\right)+c_{2} r_{2}\left(P_{g d}^{k}-X_{i d}^{k}\right)$

$X_{i d}^{k+1}=X_{i d}^{k}+V_{i d}^{k+1}$ 
where $r_{1}$ and $r_{2}$ are random number between 0 and $1, c_{1}$ and $c_{2}$ are learning factors, and $w$ is the inertia weight.

The KELM algorithm is improved by combining ACO and PSO. The steps of improving the algorithm are as follows:

(1) the parameters are initialized, and the initial KELM model is established;

(2) KELM is trained to calculate the current position and pheromone concentration of ants;

(3) the pheromone concentration of ants is updated, and the particle swarm is initialized by the best position vector;

(4) the current fitness value of particles is calculated, and $P_{\text {best }}$ is compared with $g_{\text {best }}$; if $P_{\text {best }}$ is more optimal, then it will be taken as the optimal position of swarm;

(5) the current optimal solution is recorded, and the optimal solution is output if the termination is satisfied; otherwise it returns to step (2).

\section{Case Analysis}

\section{- Experimental data and processing}

Taking the property load of two communities as the experimental subject, the historical data of community A and B between May and July were collected, including the current date, temperature and historical load. The data of May to June was used for model training, and then the data of the first day of July was used as the test sample.

Before building the model, in order to improve the reliability of the model, it is necessary to process the historical data. For incomplete data, data with load of 0 for more than two hours due to power failure and sudden change data due to special holidays, the weekly average data were used for coverage, and the calculation formula is as follows:

$$
x_{i}=\frac{x_{i-Z}+x_{i+Z}}{2} \quad Z=7 \times 24
$$

The prediction accuracy of the model was evaluated using average relative error (MAPE): MAPE $=\frac{1}{n} \sum_{i=1}^{n}\left|\frac{y_{i}^{\prime}-y_{i}}{y_{i}}\right| \times 100 \%$, where $y_{i}^{\prime}$ represents the predicted value, $y_{i}$ is the actual value, and $n$ is the number of predicted values.

\section{- Experimental results}

KELM parameter (BestC,Best $\omega$ ) obtained after being optimized by swarm intelligence algorithm was (87.2, 0.083). The improved KELM model was used for training and testing and compared with SVM and KELM models.

The prediction results are shown in Figure 1.

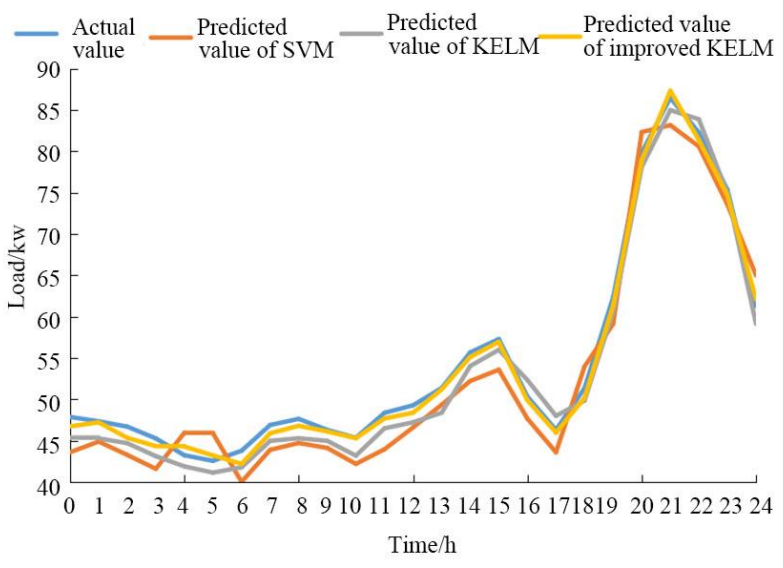

Figure 1: Prediction results of community $A$

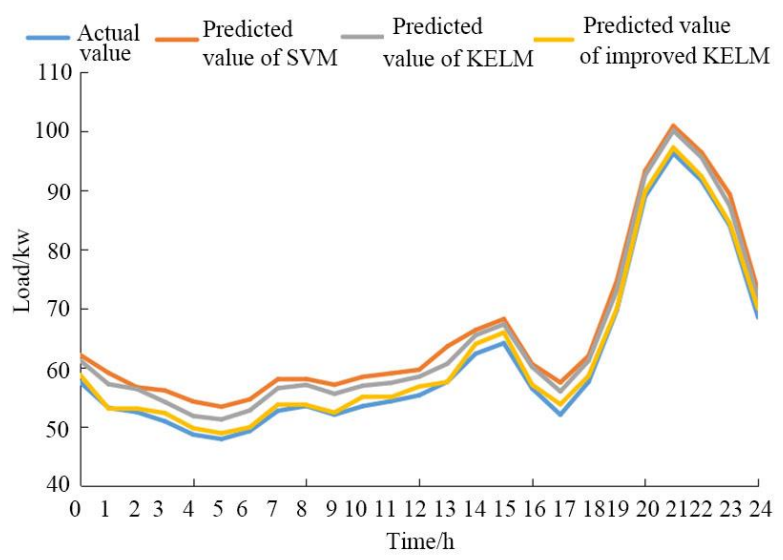

Figure 2: Prediction results of community $B$

It was seen from Figure 1 and 2 that the load had a similar trend of change within 24 hours; the load was low at 0:00 - 6:00 and then increased gradually, and the increase amplitude was large at 18:00-22:00.

Among the three prediction models, the prediction result of SVM model had the largest difference with the actual value, followed by KELM; the prediction result of the improved KELM algorithm designed in this study was the most similar to the actual value, indicating that the prediction accuracy of the improved KELM model was high.

The MAPE and running time of the three models were compared, and the results are shown in Table 1.

Table 1. MAPE and running time

\begin{tabular}{|l|l|l|l|l|}
\hline & & SVM & KELM & $\begin{array}{l}\text { Improve } \\
\text { d KELM }\end{array}$ \\
\hline \multirow{2}{*}{$\begin{array}{l}\text { Commu } \\
\text { nity A }\end{array}$} & MAPE/\% & 5.86 & 3.55 & 1.43 \\
\cline { 2 - 5 } & $\begin{array}{l}\text { Running } \\
\text { time/s }\end{array}$ & 931 & 629 & 215 \\
\hline $\begin{array}{l}\text { Commu } \\
\text { nity B }\end{array}$ & MAPE/\% & 8.35 & 5.93 & 1.57 \\
\cline { 2 - 5 } & $\begin{array}{l}\text { Running } \\
\text { time/s }\end{array}$ & 942 & 613 & 223 \\
\hline
\end{tabular}

According to the MAPE comparison of the prediction results of community A, SVM > KELM > 
the improved KELM, the MAPE of the improved KELM model was only $1.43 \%$, and the running time of the improved KELM model was also significantly shorter than SVM and KELM models. Community B also showed the same results, the MAPE of the three models were $8.35 \%, 5.93 \%$ and $1.57 \%$ respectively, the running time was $942 \mathrm{~s}, 613 \mathrm{~s}$ and $223 \mathrm{~s}$ respectively, which verified the advantages of the method in load prediction.

Taking community A as an example, the load of the first week of July was predicted by using the improved model, and the results are shown in Figure 3.

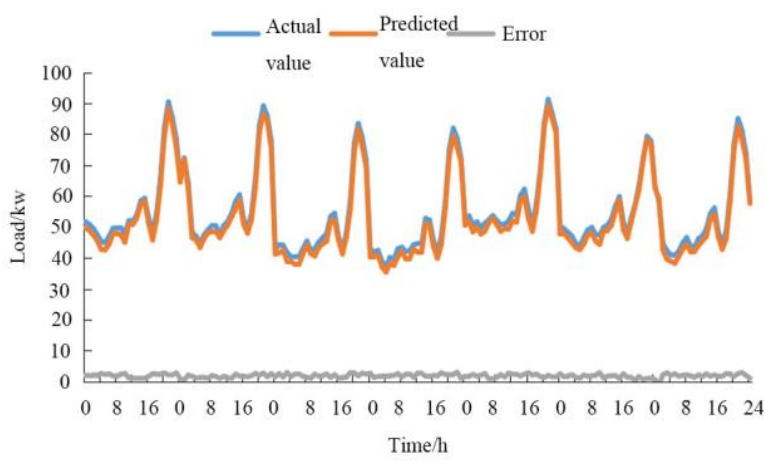

Figure 3. Prediction results in the first week of July

It was found from Figure 3 that when the improved KELM model was used for prediction, the predicted values were close to the actual values, and the error was relatively stable without large fluctuation, which showed that the method not only had good prediction accuracy, but also had good stability, i.e., it had excellent performance in load prediction.

\section{Discussion}

The progress of society has increased people's dependence on energy, and the power demand of society is also in a state of rapid growth. As one of the effective utilization modes of distributed energy [13], microgrid can operate in the grid connected or off grid mode [14], which can realize multiple links such as power generation, transmission, distribution, etc., and moreover it has function of fault self-healing and energy management; therefore it has a great potential for development. Load prediction is an important condition for the optimal operation of microgrid, and accurate load prediction plays an important role in the formulation of grid planning scheme [15]. Due to the large randomness of the micro grid power supply and the great uncertainty of the load $[16,17]$, the load prediction is difficult, so it is necessary to establish a high accuracy prediction model.

In this study, the KELM model derived from the ELM model was improved. The parameters of the KELM model were optimized by ACO and PSO.
Then the improved KELM model was used for experiments, and compared with the SVM model and KELM model.

The experimental results showed that the prediction results of the improved KELM were closest to the actual values (Figure 1 and 2), and the difference between the prediction results of SVM model and the actual values was the largest, which might be because the parameters of SVM were not optimized.

It was found from Table 1 that the MAPE value of the SVM model was the largest, followed by the KELM model and improved KELM, and the running time of the SVM model was the shortest, followed by the KELM model and improved KELM model, which showed that the improved KELM model not only had high prediction accuracy, but also had short running time, i.e., it could obtain more accurate prediction results in a short time.

The prediction results of Figure 3 also suggested that the improved KELM algorithm was reliable and worth popularizing and applying in practice.

There are still many problems to be solved in further research due to the limited capacity and time in this study although some achievements have been made in this research:

(1) the micro grid data obtained was relatively limited, only the summer load was studied;

(2) the parameter optimization of the KELM algorithm can be further improved;

(3) experiments can be carried out on load prediction of multiple environments such as factory and school.

\section{Conclusion}

In order to realize the optimal operation of microgrid, this study mainly studied its load prediction problem. The KELM algorithm was improved, and the experimental results showed that the improved algorithm was better than the SVM model and KELM model in time and accuracy, the MAPE of two communities was $1.43 \%$ and $1.57 \%$ respectively, the operation time was short, and the stability was strong; hence it has a good applicability in the load prediction of microgrid.

\section{References}

[1] Rana M, Li L. "An Overview of Distributed Microgrid State Estimation and Control for Smart Grids," Sensors, 2015, 15(2):4302-4325.

[2] Bouzid A M, Guerrero J M, Cheriti A, Bouhamida M, Sicard P, Benghanem M. A survey on control of electric power distributed generation systems for microgrid applications [J]. Renewable and Sustainable Energy Reviews, 2015, 44:751-766. 
[3] Nejabatkhah F, Li Y W. "Overview of Power Management Strategies of Hybrid AC/DC Microgrid," IEEE Transactions on Power Electronics, 2014:1-1.

[4] Nosratabadi S M, Hooshmand R A, Gholipour E. "A comprehensive review on microgrid and virtual power plant concepts employed for distributed energy resources scheduling in power systems," Renewable and Sustainable Energy Reviews, 2017, 67:341-363.

[5] Zhang X, Yu J. "Power Load Forecasting Based on Swarm Intelligence Algorithm". Journal of Computational and Theoretical Nanoscience, 2015, 12(12):5323-5332.

[6] Chang E H, Zhu G N, Chen J W. "A Combined Model Based on Cuckoo Search Algorithm for Electrical Load Forecasting,"Applied Mechanics and Materials, 2015, 737:278-282.

[7] Heng J N, Wang C, Zhao X, Wang J. "A Hybrid Forecasting Model Based on Empirical Mode Decomposition and the Cuckoo Search Algorithm: A Case Study for Power Load," Mathematical Problems in Engineering, 2016, 2016:1-28.

[8] Chitsaz H, Shaker H, Zareipour H, Wood D, Amjady N. "Short-term electricity load forecasting of buildings in microgrids," Energy and Buildings, 2015, 99:50-60.

[9] Peng H, Gu J, Hu Y, Song B. "Forecasting Model for Saturated Load Based on Chaotic Particle Swarm Optimization-Gaussian Process Regression," Dianli Xitong Zidonghua/Automation of Electric Power Systems, 2017, 41(21):25-32 and 155.

[10] Wei S, Mohan L. "Application of improved artificial neural networks in short-term power load forecasting," Journal of Renewable and Sustainable Energy, 2015, 7(4):043106.

[11] Guo X, Guo X, Su J. "Improved Support Vector Machine Short-term Power Load Forecast Model Based on Particle Swarm Optimization Parameters," Journal of Applied Sciences, 2013, 13(9):1467-1472.

[12] Tang J, Deng C, Huang G B. "Extreme Learning Machine for Multilayer Perceptron," IEEE Transactions on Neural Networks \& Learning Systems, 2017, 27(4):809-821.

[13] Rezvani A, Izadbakhsh M, Gandomkar M. "Microgrid dynamic responses enhancement using artificial neural network-genetic algorithm for photovoltaic system and fuzzy controller for high wind speeds,". International Journal of Numerical Modelling: Electronic Networks, Devices and Fields, 2016, 29(2):309332.

[14] Sohn J M. “Generation Applications Package for Combined Heat Power in On-Grid and Off-Grid Microgrid Energy Management System," IEEE Access, 2016:1-1.

[15] Dou C, Zheng Y, Yue D, Zhang Z, Ma K. "Hybrid model for renewable energy and loads prediction based on data mining and variational mode decomposition," IET Generation, Transmission \& Distribution, 2018, 12(11):2642-2649.

[16] Khodaei A, Bahramirad S, Shahidehpour M. "Microgrid Planning Under Uncertainty," IEEE Transactions on Power Systems, 2015, 30(5):2417-2425.

[17] Ding M, Chu M, Pan H, Xu Z C. "Operation optimization modeling and uncertainty analysis for hybrid AC/DC microgrids," Automation of Electric Power Systems, 2017, 41(5):1-7 and 45. 